\title{
Improving Posture Accuracy of Non-Holonomic Mobile Robot System with Variable Universe of Discourse
}

\author{
Siti Nurmaini ${ }^{1}$, Bambang Tutuko ${ }^{2}$, Kemala Dewi $^{3}$, Velia Yuliza ${ }^{4}$, Tresna Dewi $^{5}$ \\ ${ }_{1,2,3,4}$ Computer Engineering Department, Universitas Sriwijaya \\ ${ }^{5}$ Electrical Engineering Department, Politeknik Negeri Sriwijaya \\ Corresponding author, e-mail: sitinurmaini@gmail.com, beng_tutuko@yahoo.com, \\ kemaladewi09@gmail.com, valiza47@yahoo.com, tresna_dewi@polsri.ac.id
}

\begin{abstract}
This paper presents a method to decrease imprecision and inaccuracy that have the tendency to influence the posture of non-holonomic mobile robot by using the adaptive tuning of universe of discourse. As such, the primary objective of the study is to force the posture error of $\boldsymbol{x}(\boldsymbol{t}), \boldsymbol{y}(\boldsymbol{t})$, and $\boldsymbol{\theta}(\boldsymbol{t})$ towards zero. Hence, for each step of tuning the fuzzy domain, about $20 \%$ of imprecision and inaccuracy had been added automatically into the variable universe fuzzy, while the control input was bound via scaling gain. Furthermore, the simulation results showed that the tuning of universe fuzzy parameters could increase the performance of the system from the aspects of response time and error for steady state through better control of inaccuracy. Besides, the domains of universe fuzzy input [-4,4] and output $[0,6]$ exhibited good performance in inching towards zero values as the steady state error was about $1 \%$ for $x(t)$ position, $0.02 \%$ for $y(t)$ position, and $0.16 \%$ for $\theta(t)$ orientation, whereas the posture error in the given reference was about $0.0002 \%$
\end{abstract}

Keywords: Non-holonomic system, Kinematic model, Fuzzy logic controller, Posture error, Variable universe of discourse

Copyright $\odot 2017$ Universitas Ahmad Dahlan. All rights reserved.

\section{Introduction}

The control of mobile robot can be modeled in several conducts. However, the most significant aspects that should be considered in to determine a model are the complexity and its use. In the designs of mobile robot can be divided into two: holonomic and non-holonomic systems. Holonomic robot refers to the robot in which the amount of controllable inputs is similar to the controllable level of autonomy [1]. Meanwhile, the non-holonomic robot has restricted mobility due to lower amount of controllable inputs, thus posing a more pressing issue, when compared to that of holonomic robot [2]. Therefore, several methods have been suggested to obtain the best solutions for optimum performance in relation to control. Moreover, the control strategies of mobile robot can be classified into two types: closed and open loop controls. As for the control with open loop type, the inputs, which are comprised of the initial knowledge and the last speck of it (inclusive of the paths desired among them for adherence of path), are precalculated for mobile robots (torques/velocities). Unfortunately, such approach can still be affected by interruptions and errors. Nonetheless, the closed loop technique is unaffected by errors because its inputs are derived from the whole system, instead of just the beginning and ending points. Hence, any error or interruption that occurs generates deviances away from the assumptions, mainly due to the inputs. Nevertheless, close loop controller design has an issue; computing complexity in non-holonomic robot, chiefly because of the non-linear equations applied in both kinematic and dynamics systems.

In fact, non-holonomic mobile robots come in three main categories to as certain that the controller functions well in the system: 1) based on kinematic model [3-7], 2) based on dynamic model [8-14], and 3) based on both kinematic and dynamic models [15-19]. These models have their own limitation and complexity. Several researchers have looked into these issues and developed several controllers, including adaptive feedback [20], back stepping [21], feedback linearization [22], and sliding mode [23-29]. Sliding mode control and its features are the good options to compensate mobile robots under actuated system. Mobile robot is said to have the under-actuated system since two inputs (velocities) are used to control three outputs 
(positions) and this constraint also contributes in adding the complexity of the system [24-29]. However, the drawbacks of these approaches are that a tedious analysis is required to design the control algorithms and tuning the parameters is indeed a complicated task. These are especially true for trajectory control, where the controller algorithm is designed with information extracted from the reference path. In fact, most theoretical aspects in the systems developed for tracking via trajectory have dismissed the instabilities and the inaccuracies that might occur from the practical stance [24-32]. Such dismissal of negativities actually could lead to increased steady state error and lack in transience. Hence, the selected methods for controlling trajectory could be enhanced with the inclusion of external disturbances and model inaccuracies.

Furthermore, the advances in computational resources have made developing a mobile robot with a control system that is fully autonomous a possible feat [27]. With that, a number of issues related to control have been ironed out with the development of several control systems, for instance, neural network [9], [34-35] and fuzzy control [4-5], [8], [10-11]. Meanwhile, for issues related to those nonlinear, numerous analyses have highlighted the benefits of fuzzy controller, for example, its unaffected performance with presence of noise. Its application in an intricate structure where the dynamic model is vague or unavailable, excellent in overcoming error related to nonlinear issues, as well as the noted enhanced sturdiness in the system of a robot [4-5], [10-11], [12, 14]. The linguistic data employed by fuzzy logic controller offer some benefits, for instance, rule-based algorithm, robustness, general approximation theorem, and model-free. Moreover, the fuzzy controller generates a system that transforms the strategy of linguistic control from the knowledge of expert to a strategy that is automatic [11-12], [36]. Hence, the inaccuracies controller that is designed is incorporated into the function of membership. Therefore, the techniques of fuzzy control have sparked more interest in addressing issues related to non-holonomic mobile robots control.

The universe of discourse has to be appropriate designed in developing a fuzzy logic control because it is a significant aspect in determining the performance of the fuzzy controller. It defines the interval of all possible values or the set of higher and lower confines for the set of fuzzy employed in determining the aspects of variables for fuzzy. Moreover, the sets of universe of discourse are represented as uncertainty in input and output membership functions (MFs), with varied grades of membership in the interval $[0,1]$. In specifying the universe of discourse, the first step is determining an appropriate range that should be weighed in seriously to identify the features of the selected variables in the designed scheme. Moreover, the data would become off scale if the range were set to be narrow, thus affecting the performance of the system. On the contrary, a larger range generates wider MFs on either right or left, thus leading to exaggerated values in input. Besides, suitable interval value for the universe of discourse in the fuzzy control process could produce positive response in both transient and steady state error due to the elaboration of imprecision, inaccuracy, and uncertainty in mobile robot $[37,38,39]$. However, determining the value is definitely challenging. Therefore, the best scaling factors of fuzzy universe of discourse is desirable.

In this paper, all possibilities of universe of discourse had been analyzed with simple fuzzy controller algorithm to determine the response of steady state error. As such, the analysis of universe of discourse had been employed to design the MFs of fuzzy controller. In addition, the universe of discourse changes as the error change, especially with fixed amount control rules. Therefore, the rules of the control are regulated in a dynamic manner. The rule-based and the fuzzy inference systems exploit the information related to the reference and the actual path ahead of the mobile robot, as well as the distance of the next position for the robot to safely drive to the target with minimal error trajectory. The output of the fuzzy control refers to the maximum values of angular and linear velocities; in which the motion references of mobile robot satisfy the bounds of velocities and accelerations. This method of adaptive tuning of universe of discourse is set to be the novelty of this paper to decrease imprecision and inaccuracy that have the tendency to influence the posture of a mobile robot that suffers the non-holonomic constraint. The feasibility of the proposed method is proven with simulation, based on a real robot performing its task in an unknown environment. 


\section{Research Method}

\subsection{Non-holonomic System}

A non-holonomic differential drive mobile robot (DDMR) had been considered in this paper. Such robotic system must control the error tracking system because the DDMR operation had two left and right drive wheels mounted on similar axis, while another two free wheels were installed on the back wheels. The robot was driven separately and only moves in a horizontal plane had been taken into account, while any non-holonomic constriction was enforced upon the kinematics. This is because; the kinematic model describes the restrictions equation, which dismisses symbolic integration in determining explicit correlations between the positions of robot at global and local frames of coordinates. The 2D model of DDMR mobile robot, which was considered to move in Cartesian plane, is shown in Figure 1.

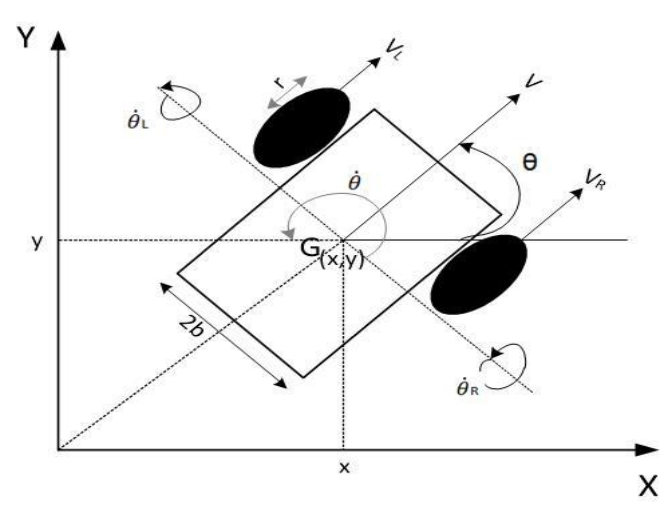

Figure 1. DDMR model in 2D Cartesian plane

In order to analyze the system and to develop the tracking controller, a differential drive represented the model of kinematics for mobile robot. In fact, the governing equations of the kinematic model, inclusive of non-holonomic constraints, are rather well known (see [10], [1315] for detailed derivation). Figure 1 shows all the variables used in the kinematic model, while the definitions are discussed in the following:

$2 b$ distance of the robot from center of right wheel to left wheel

$r$ radius of each wheels

$\theta$ robot orientation

$v$ robot linear velocity

$\omega$ angular velocity of the robot

$V_{R}$ right wheel linear velocity

$V_{L}$ left wheel linear velocity

Furthermore, as the analysis of castor-free had been disregarded, the mobile robot configuration is categorized into three general variables $q(t)$ to describe the point for robot, as well as the control input $u(t)$, as defined in the following matrix:

$$
q(t)=[x(t), y(t), \theta(t)]^{T} \text { and } u(t)=\left[\dot{\theta}_{R}(t), \dot{\theta}_{L}(t)\right]^{T}
$$

The values of right and left wheels in angular velocity are:

$$
\dot{\theta}_{R}(t)=\frac{1}{2 \pi r} \cdot v_{R}(t) \text { and } ; \dot{\theta}_{L}(t)=\frac{1}{2 \pi r} \cdot v_{L}(t),
$$

and the translational velocities of the both wheels are given in the following:

$$
v_{R}(t)=v(t)+b \dot{\theta}(t) \text { and } v_{L}(t)=v(t)-b \dot{\theta}(t)
$$

From equations (2) and (3), a linear velocity is $v(t)$ and an angular velocity is $\dot{\theta}(t)$, as follows: 


$$
v(t)=\frac{1}{2}\left(v_{R}(t)+v_{L}(t)\right) ; \dot{\theta}(t)=\frac{v_{R}(t)-v_{L}(t)}{2 b}
$$

However, the DDMR is limited in terms of movement due to non-holonomic constrain with two primary assumptions. The first assumption is no lateral slip motion, which refers to the movement of the robot in a curved motion (forward and backward), but not sideward. The velocity at the midpoint of both wheels $(G)$ was obtained from Figure 2(b), and the related equation is given below:

$$
v(t)=\dot{x}(t) \cos \theta(t)+\dot{y}(t) \sin \theta(t)
$$

Besides, as for the frame of robot, the value of velocity for the wheel at the locus point, $G$, is zero, along the lateral axis as it only moves along $x$ axis and $y$ axis [7]. Therefore the velocity equation is:

$$
\dot{x}(t) \sin \theta(t)-\dot{y}(t) \cos \theta(t)=0
$$

Meanwhile, the second assumption reflects the pure rolling constraint because the wheels did not slip on the floor and thus, portrayed a limitation of pure rolling. With that, the following depicts the linear velocity for both right and left wheels:

$$
v_{R}(t)=r \dot{\theta}_{R}(t) \text { and } v_{L}(t)=r \dot{\theta}_{L}(t)
$$

By looking at the starting position of the wheel to the Cartesian field, $x y$ can be obtained from the equation of position wheeled robot, $x(t)=v(t) \sin \theta(t), y(t)=v(t) \cos \theta(t)$, and $\theta(t)=v_{\theta}(t)$. Thus, the transformation matrix equations from the initial position can be defined as:

$$
T_{N H}(q)=\left[\begin{array}{cc}
\frac{r}{2} \cos \theta(t) & \frac{r}{2} \cos \theta(t) \\
\frac{r}{2} \sin \theta(t) & \frac{r}{2} \sin \theta(t) \\
\frac{r}{2 b} & -\frac{r}{2 b}
\end{array}\right]
$$

Furthermore, the velocity of the robot is defined as the control input $u(t)$ and it consists of three parameters: $u_{1}(t)=\dot{\theta}_{R}(t), u_{2}(t)=\dot{\theta}_{L}(t)$, and $\dot{\theta}(t)=\omega(t)$. From equation (8) and three parameters of input equation, the model of DDMR kinematic equation is: $\dot{q}(t)=T_{N H}(q) u(t)$ or,

$$
\left[\begin{array}{c}
\dot{x}(t) \\
\dot{y}(t) \\
\dot{\theta}(t)
\end{array}\right]=\left[\begin{array}{cc}
\cos \theta(t) & 0 \\
\sin \theta(t) & 0 \\
0 & 1
\end{array}\right]\left[\begin{array}{c}
v(t) \\
\omega(t)
\end{array}\right]
$$

\subsection{Posture Error Modeling}

The error model of DDMR was obtained by reducing the reference position with the actual position of the robot. As such, two postures had been employed for this control scheme: reference and current postures, as described in the following two equations:

$$
\begin{aligned}
& q_{r}(t)=\left[x_{r}(t), y_{r}(t), \theta_{r}(t)\right]^{\top}, \\
& q_{c}(t)=\left[x_{c}(t), y_{c}(t), \theta_{c}(t)\right]^{\top}
\end{aligned}
$$

Reference posture refers to the goal posture for a mobile robot, which is defined as imaginary robot and it strictly adheres to reference path, whereas current posture reflects the original posture, as illustrated in Figure 2. On the other hand, posture error is defined as $q_{e}(t)$ and represented by $q_{e}(t)=\left[e_{x}(t), e_{y}(t), e_{\theta}(t)\right]^{T}$, while $e_{x}(t)$ is the error in driving direction, $e_{y}(t)$ is the error in lateral direction, and $e_{\theta}(t)$ refers to error related to robot orientation. In addition, $q_{e}(t)$ reflects the transformation result between the reference position $q_{r}(t)$ in a local 
coordinate system with the previous position, $q_{c}(t)$, and $X$ axis in the direction towards the robot is $\theta_{c}(t)$ (Figure 3). The posture error is defined in Equation (12):

$$
q_{e}(t)=T_{e}\left(q_{r}(t)-q_{c}(t)\right)
$$

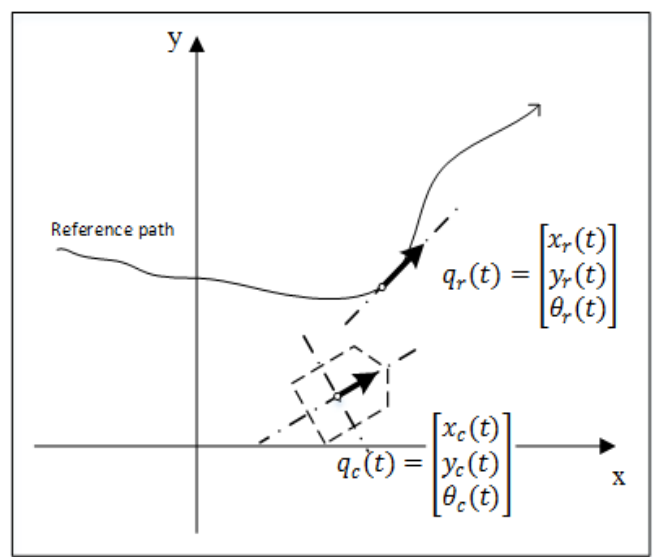

Figure 2. Mobile robot posture

$T_{e}(t)$ found in equation (12) refers to the rotation matrix as seen from space 3D Cartesian.

$$
T_{e}(\mathrm{t})=\left[\begin{array}{l}
x(t) \\
y(t) \\
\theta(t)
\end{array}\right]=\left[\begin{array}{ccc}
\cos \theta(t) & \sin \theta(t) & 0 \\
-\sin \theta(t) & \cos \theta(t) & 0 \\
0 & 0 & 1
\end{array}\right]
$$

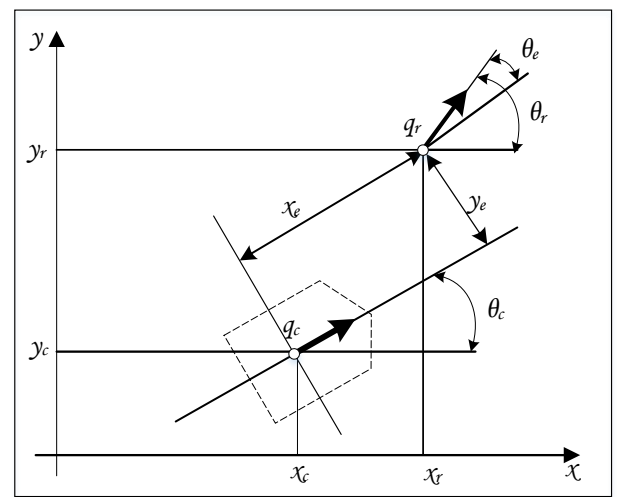

Figure 3. Error posture

The equation of error is defined by substituting Equation (13) with Equation (12), as shown in Equation (14):

$$
\begin{aligned}
& q_{e}(t)=\left[\begin{array}{l}
e_{x}(t) \\
e_{y}(t) \\
e_{\theta}(t)
\end{array}\right]=\left[\begin{array}{ccc}
\cos \theta_{C}(t) & \sin \theta_{C}(t) & 0 \\
-\sin \theta_{C}(t) & \cos \theta_{C}(t) & 0 \\
0 & 0 & 1
\end{array}\right]\left[\begin{array}{lll}
q_{R}(t) & q_{C}(t)
\end{array}\right] \text { or } \\
& {\left[\begin{array}{l}
e_{x}(t) \\
e_{y}(t) \\
e_{\theta}(t)
\end{array}\right]=\left[\begin{array}{ccc}
\cos \theta(t) & \sin \theta(t) & 0 \\
-\sin \theta(t) & \cos \theta(t) & 0 \\
0 & 0 & 1
\end{array}\right]\left[\begin{array}{l}
x_{r}(t)-x_{c}(t) \\
y_{r}(t)-y_{c}(t) \\
\theta_{r}(t)-\theta_{c}(t)
\end{array}\right]}
\end{aligned}
$$


In order to determine the change of error $(\Delta e(t))$ or $\left(\Delta e_{x}(t) ; \Delta e_{y}(t) ; \Delta e_{\theta}(t)\right)$, one can calculate $e(t)-e(t-1)$ ). This value becomes an input for fuzzy controller, while the equation is described in Equation (15)-(17),

$$
\begin{aligned}
& \Delta e_{x}(t)=e_{x}(t)-e_{x}(t-1) \\
& \Delta e_{y}(t)=e_{y}(t)-e_{y}(t-1) \\
& \Delta e_{\theta}(t)=e_{\theta}(t)-e_{\theta}(t-1)
\end{aligned}
$$

Taking robot kinematics in Equation (9) and differentiating Equation (14), the postureerror model is given as:

$$
\left[\begin{array}{c}
\dot{e}_{x}(t) \\
\dot{e}_{y}(t) \\
\dot{e}_{\theta}(t)
\end{array}\right]=\left[\begin{array}{cc}
\cos \dot{e}_{\theta}(t) & 0 \\
\sin \dot{e}_{\theta}(t) & 0 \\
0 & 1
\end{array}\right]\left[\begin{array}{c}
v_{r}(t) \\
\omega_{r}(t)
\end{array}\right]+\left[\begin{array}{cc}
-1 & e_{y}(t) \\
0 & -\dot{e}_{x}(t) \\
0 & -1
\end{array}\right] u
$$

where $v_{r}(t)$ and $\omega_{r}(t)$ are the reference translational and angular velocity respectively, whereas $u(t)$ reflects the input for control given in Equation (9).

\subsection{Controller Design}

\subsubsection{Fuzzy Logic System}

The fuzzy logic system is developed completely with fuzzy sets, comprising of four components: fuzziffer, rule base, inference engine, and defuzziffier (Figure 4). In a fuzzy set, all elements that belong to it have a particular membership level. Hence, an assumption may be false or true, or it could be partially true (or partially false) at certain degree. Such degree of membership is known as membership value, which is valued in the range of zero to one. The mapping between elements of a fuzzy sets and its membership value is given by MFs. Other than that, fuzzy sets (FSs) refers to the generalization of a crisp set, whose membership grades can only be 0 or 1 . A fuzzy set of $A$ is defined on universe of discourse $X$ and it is featured using membership grade $\mu_{A}(x)$ that incorporates values from interval $[0,1]$.

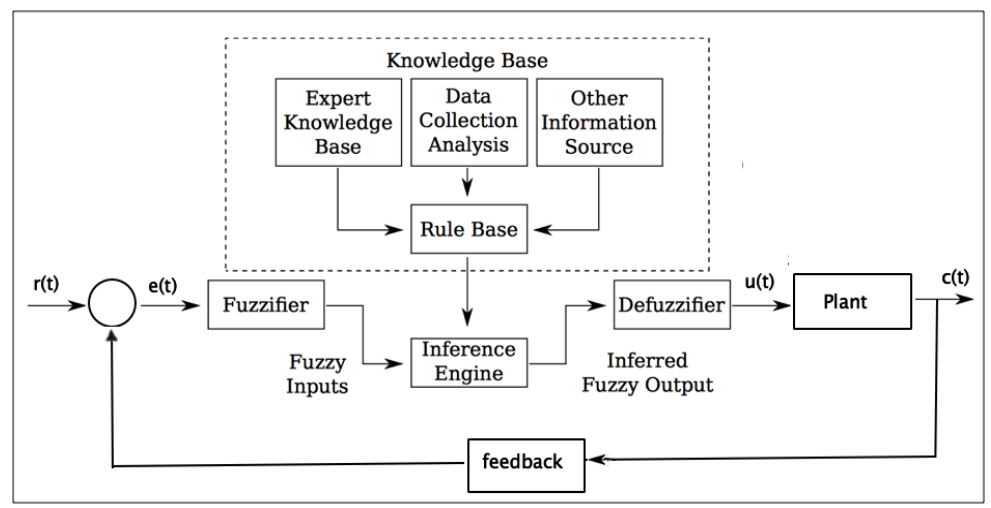

Figure 4. Fuzzy Logic Controller (FLC)

The following defines fuzzy sets $A$ over universe of discourse $X[34]$ :

$$
\mu_{A}(x): X \rightarrow\{0,1\}
$$

With that, two aspects are generated for universe $X$ in relation to the $\mathrm{x}$ element for the universe of discourse, in which the characteristic function of $X, \mu_{A}(x)$, becomes equivalent to 1 , when set $A$ has the element of $x$, but it becomes 0 if otherwise. Furthermore, the following expresses fuzzy sets $\mathrm{A}$ at a condition when the universe of discourse of $X$ becomes continuous: 


$$
A=\int_{X} \mu_{A}(x) / x
$$

Here $\int$ does not denote integration, but accumulation of the overall points $x \in X$ linked to membership grade, $\mu_{A}(x)$. Meanwhile, when the universe of discourse $X$ is discrete and finite, the fuzzy sets of $A$ may be expressed as in the following:

$$
A=\sum_{i=1}^{n} \frac{\mu_{A}\left(u_{i}\right)}{u_{i}}
$$

Besides, a mapping is generated by fuzziffer with input of crisp $x=\left(x^{1}, \ldots, x^{p}\right)$ into fuzzy sets in $U$. These fuzzy sets are associated with terms that appear in the antecedents or consequents of rules, as well as with inputs to and output of the fuzzy logic system (FLS). However, in practical, it is rather challenging to identify the plant model, which is rather commonly found as nonlinear. Hence, the use of FLC overcomes this error. However, most cases related to fuzzy universe cannot be defined in a manual manner as commendably, thus demands tuning. Furthermore, an apt value of the universe of discourse can improve the rate of accuracy in a DDMR system.

In fact, the tuning strategy is not difficult for implementation with the automatic tuning approach. The main goal of this strategy is to improve the performance of steady state error, and therefore, the scaling factor is generated to ascertain the stable close loop, as well as the rapid convergence of the control strategy. In addition, the scaling factor is developed closely based on the desired performance. The parameters $e(t)$ and $\Delta e(t)$, which act as inputs to the FLC, function as the scaled values of error. The scaling factor, moreover, is connected to the behavior of control, for instance, i) the large value of $e(t)$ can increase the band around the values of steady state error, thus causing limited cycle oscillations and instability, ii) the minute value of $\Delta e(t)$ enhances the overshoot and causes instability, and lastly, iii) in achieving satisfactory steady state error, $\Delta e(t) \geq e(t)$ must be satisfied, where $e_{s(t)}$ and $\Delta e_{s}(t)$ function as scaled values of error by using upper and lower bounds. As such, a piece wise linear function $S$ can be employed to limit the bound of gain $G$. This control variable $u$ is updated with the following equation: $\mathrm{u}(\mathrm{t})=\mathrm{u}(\mathrm{t}-1)+\mathrm{G}^{*} \Delta u_{f}$, where $\mathrm{u}(\mathrm{t})$ is the actual value of error, $\mathrm{u}(\mathrm{t}-1)$ is the value of sample, $G$ is the scaling factor, and $\Delta u_{f}$ is the output of fuzzy controller.

\subsubsection{Trajectory Tracking Controller}

The structure of fuzzy logic controller for trajectory tracking has three inputs, which are $e_{x}(t)$ and $e_{y}(t)$ that function as the positions of errors, as well as $e_{\theta}(t)$ that is denoted as orientation error, which is found between the real value and the reference value. Meanwhile, $v(t)$ and $\omega(t)$ are known as the outputs, in which $v(t)$ reflects the robot centroid velocity, whereas $\omega(t)$ refers to that of angular velocity. Moreover, it is vital to have simplified rules for a reasonable computing time. Figure 5 illustrates a block diagram of fuzzy controller.

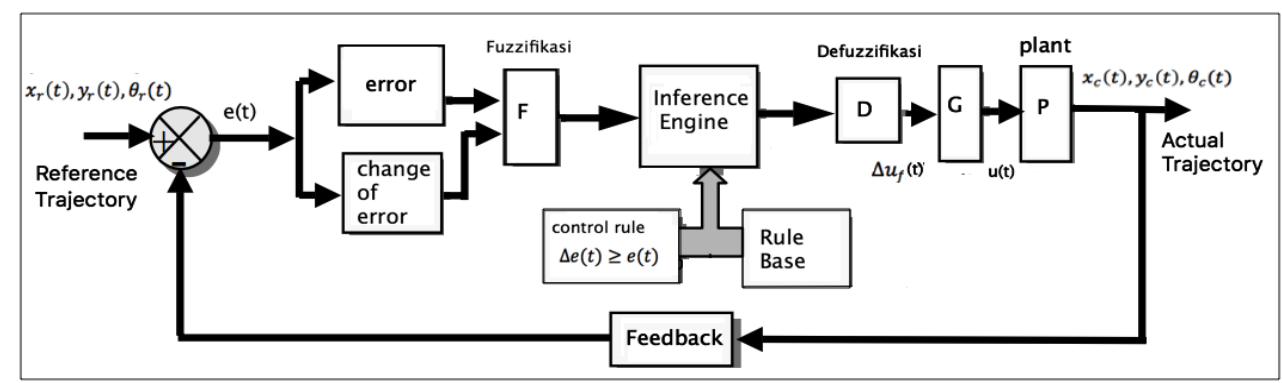

Figure 5. Fuzzy logic controller with auto tuning

In this study, the input of MFs to the fuzzy controller is error and change of error (position errors $(x(t), y(t))$, and orientation error $\theta(t))$. Such values are computed to display the 
performance of a controller when the errors are compensated in terms of time response and steady state error.

The triangular shapes found in Figure 5 reflect the MFs, while the initial universe of discourse is normalized in $[-1,1]$ for input, and $[0,6]$ for output by using appropriate scale factors. In addition, Figures 6(a), (b), (c), and (d) depict the input with Negative, Zero, and Positive fuzzy sets, respectively, as well as the output MFs notations in Slow, Medium, and Fast modes.

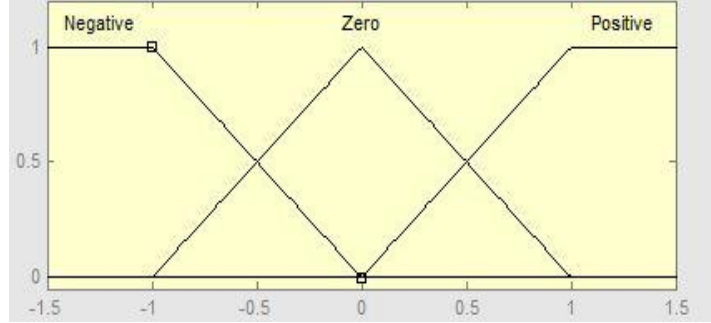

(a) Error e(t)

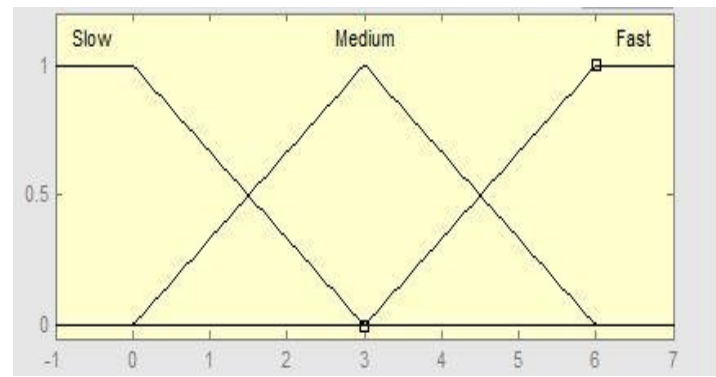

(c) Linear velocity $v(t)$

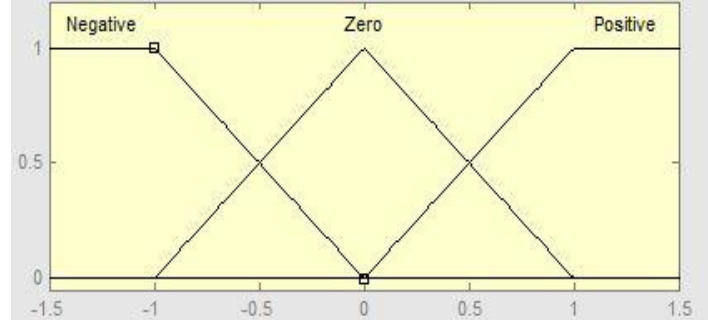

(b) Change of error $\Delta e(t)$

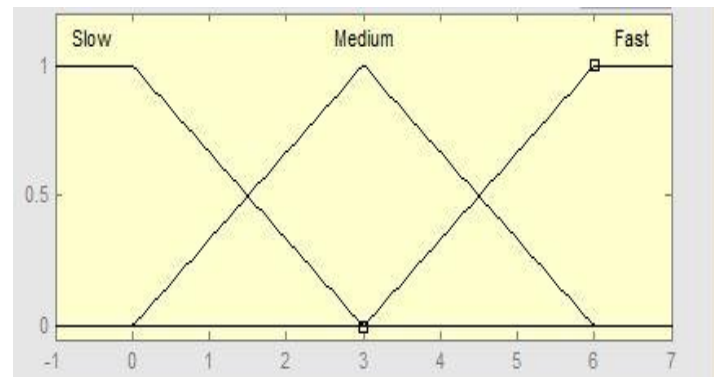

(d) Angular velocity $\omega(t)$

Figure 6. Universe of discourse fuzzy sets

Furthermore, this paper adopted 18 rules as the base set of Fuzzy controller that governed the correlations of input-output for fuzzy controller precisely by adhering to the inference engine of Mamdani style. With two output signals controlled, the fuzzy rule bases are as designed in Table 1.

Table 1. Fuzzy rule bases for $v(t)$ and $\omega(t)$

\begin{tabular}{|c|c|c|c|}
\hline$\Delta e(\mathrm{t})^{e(t)}$ & Negative & Zero & Positive \\
\hline Negative & $\begin{array}{l}\mathrm{v}(\mathrm{t}) \text { : slow } \\
\omega(t) \text { : slow }\end{array}$ & $\begin{array}{l}\mathrm{v}(\mathrm{t}): \text { medium } \\
\omega(t): \text { medium }\end{array}$ & $\begin{array}{l}\mathrm{v}(\mathrm{t}) \text { : slow } \\
\omega(t) \text { : slow }\end{array}$ \\
\hline Zero & $\begin{array}{l}\mathrm{v}(\mathrm{t}) \text { : medium } \\
\omega(t) \text { : medium }\end{array}$ & $\begin{array}{l}\mathrm{v}(\mathrm{t}) \text { : fast } \\
\omega(t): \text { fast }\end{array}$ & $\begin{array}{l}\mathrm{v}(\mathrm{t}) \text { : medium } \\
\omega(t) \text { : medium }\end{array}$ \\
\hline Positive & $\begin{array}{l}\mathrm{v}(\mathrm{t}) \text { : slow } \\
\omega(t) \text { : slow }\end{array}$ & $\begin{array}{l}\mathrm{v}(\mathrm{t}): \text { medium } \\
\omega(t): \text { medium }\end{array}$ & $\begin{array}{l}\mathrm{v}(\mathrm{t}) \text { : slow } \\
\omega(t) \text { : slow }\end{array}$ \\
\hline
\end{tabular}

In determining if the proposed fuzzy logic controller is indeed effective in overcoming trajectory tracking error, a number of control simulations were performed. As such, the tracking controller, as depicted in (1) for mobile robot, was investigated via simulations. The initial position of the robot was assumed to be $\mathrm{q}(0)=[0,0,0.78]$, while reference position was $\mathrm{q}($ act $)=$ $[3,3,0.78]$, and initial velocity at $v(0)=[0,0]$. Figure 7 presents the performance of mobile robot when operated without fuzzy controller, depicting that the response of error position $(x(t), y(t))$ and the error orientation in close loop condition had been without any controller. 


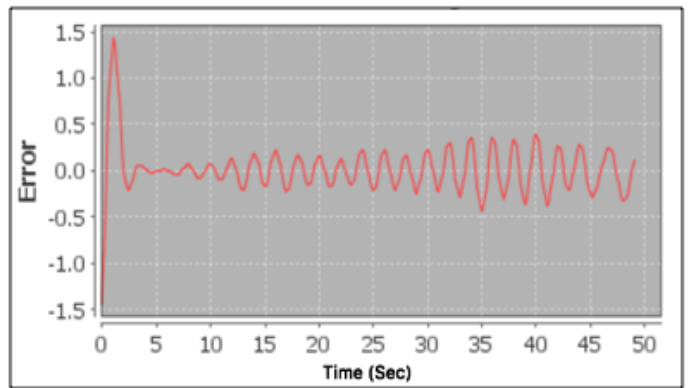

(a) Position error of $x(t)$

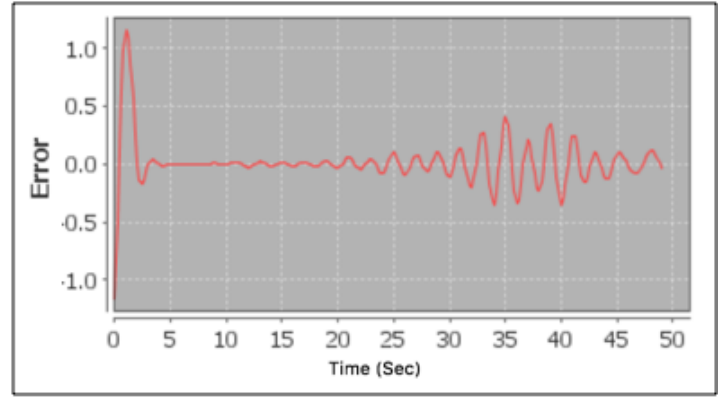

(b) Position error of $y(t)$

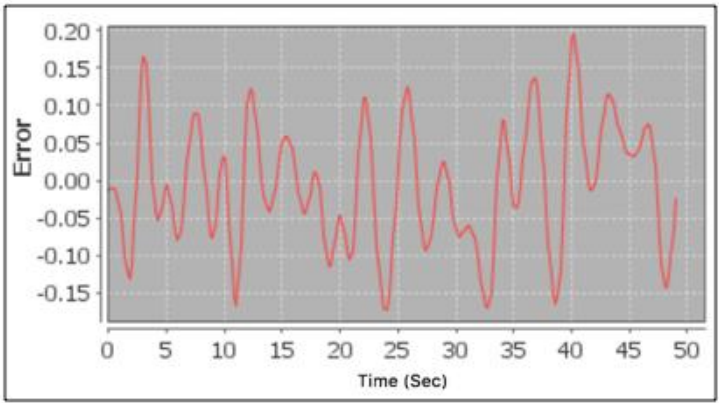

(c) Orientation error of $\theta(t)$

Figure 7. Posture errors of DDMR without controller

In order to overcome the bad response of actuator when control signals $v(t)$ and $\omega(t)$ are produced, three experiments had been initiated. Basically, in tracking the error position to zero, the fuzzy controller must be determined by the bound of uncertainty. This is because; the value of uncertainty bound determines the accuracy and the stability of the controller, mainly due to the uncertainties and noise produced by sensor, actuator, and the environment. These uncertainties make determining the antecedents and the consequent fuzzy MFs a challenging task. While MFs are determined and tuned in certain environmental conditions, modification to other environments might be needed. Thus, as for this study, the number of input and output for MFs were not changed, but the uncertainty bounds for input and output of MFs were changed. In cases 1 and 2 , the input uncertainty increased from $[-1,1]$ to $[-4,4]$, while in case 3 , the output uncertainty bound increased from $[0,6]$ to $[0,10]$. The results obtained from the experiments were compared especially to ascertain that the controller did track the posture error close to zero.

\section{Case 1: FLC with 3 MFs, where inputs of MFs were bound by $[-1,1]$ and outputs of MFs were bound by $[0,6]$}

In the first experiment, both input and output of MFs were determined from the prior experiment. Thus, the fuzzy controller output must decrease the errors of position and orientation to zero. From the experimental results, all errors of position and orientation are illustrated in Figure 8(a), (b), and (c), respectively. The findings showed that the errors indeed neared zero, in which the tracking of the trajectory had been near the anticipated value, while errors of velocity decreased to a smaller value. Nonetheless, Figure $8(\mathrm{~d})$ portrays that the trajectory tracking failed to hit the target zero, as the error tracking increased after $1.0 \mathrm{sec}$. Furthermore, the fuzzy controller did not display adequate control signal to the actuator, thus led to instability after $1.0 \mathrm{sec}$. Besides, the fuzzy controller with fuzzy sets of universe of discourse between $[-1,1]$ and $[0,6]$ did not represent the imprecision and inaccuracy as uncertainty in input and output of MFs. Therefore, for $1.0 \mathrm{sec}$ and above, the errors of trajectory grew larger chiefly because the steady state errors could not be minimized. 


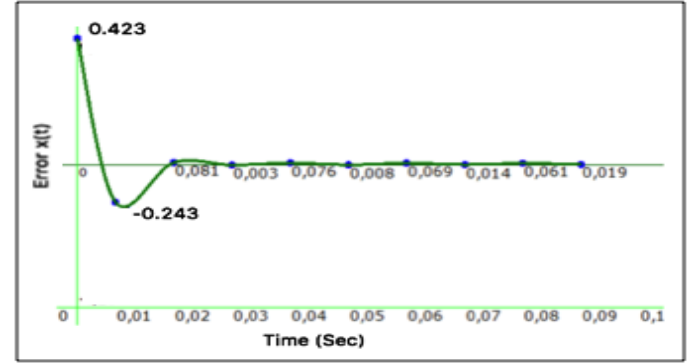

(a) Position error of $\mathrm{x}(\mathrm{t})$

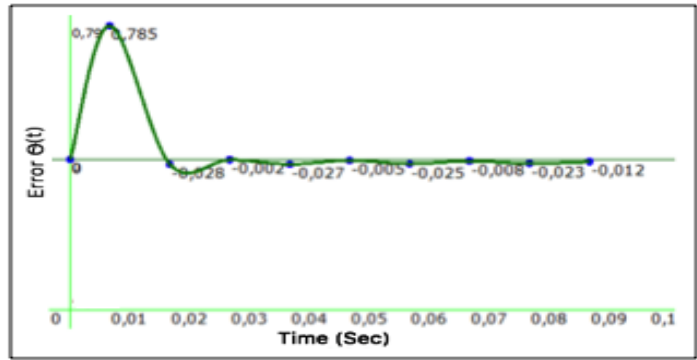

(c) Orientation error of $\theta(t)$

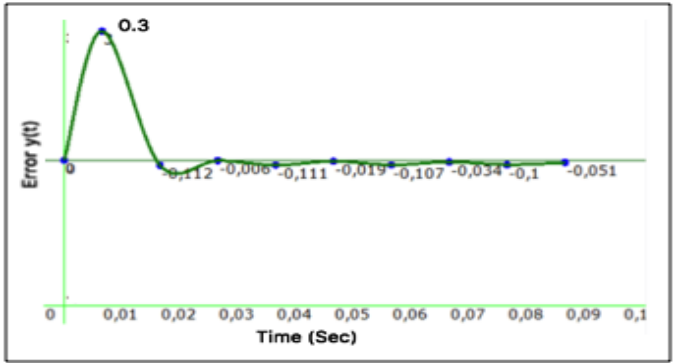

(b) Position error of $y(t)$

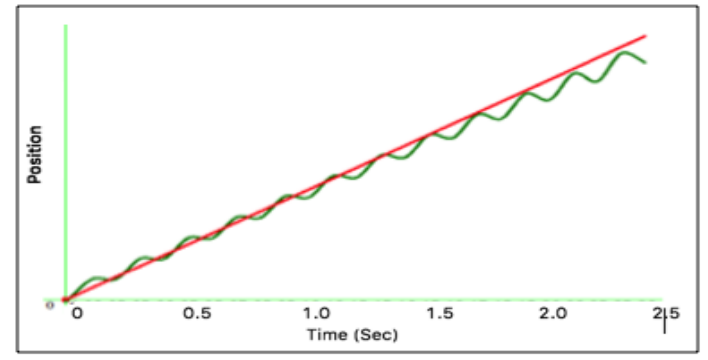

(d) Posture error of DDMR

Figure 8. Steady state errors of trajectory tracking with FLC 1

\section{Case 2: FLC with 3 MFs, where inputs of MFs were bound by $[-2,2]$ and outputs of MFs were bound by $[0,6]$}

The fuzzy sets were changed from $[-1,1]$ to $[-2,2]$, in order to cover imprecision and inaccuracy for inputs of MFs, while retaining the outputs of MFs in the second experiment. Figures 9(a), (b), and (c) illustrate the results retrieved for errors of position and orientation, whereas Figure $9(\mathrm{~d})$ describes the motion exerted by mobile robot in tracking the related reference. Therefore, by employing the fuzzy sets in such domain, the errors of steady state for $x(t), y(t)$, and $\theta(t)$ had been small and closer to the reference. However, the response time of error had been slower and the error failed in hitting the zero value. Besides, the average of steady state error was about 2 - $5 \%$ from the reference.

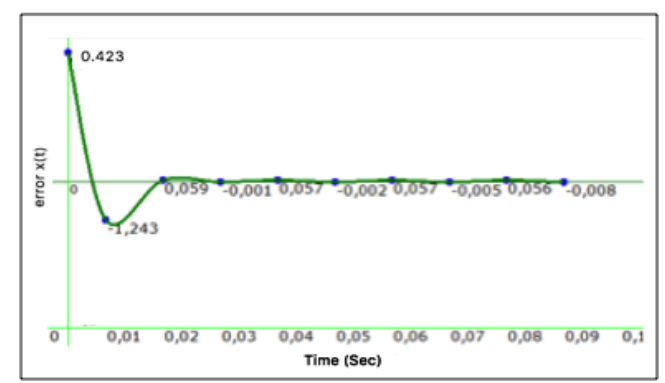

(a) Position error of $x(t)$

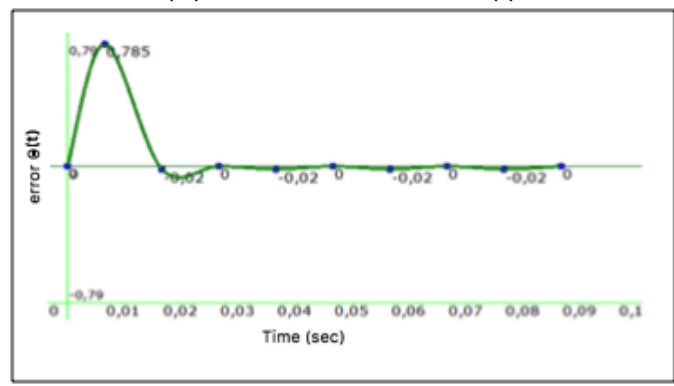

(c) Orientation error of $\theta(t)$

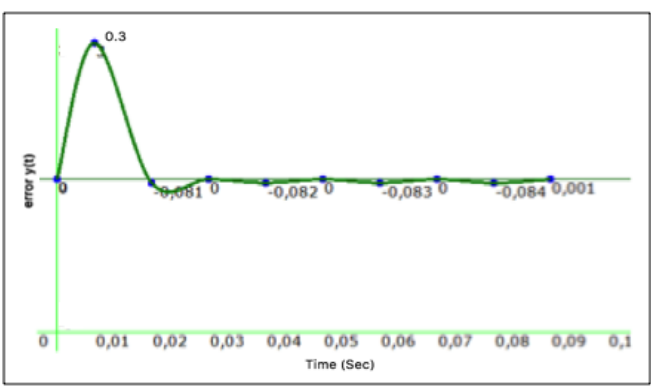

(b) Position error of $y(t)$

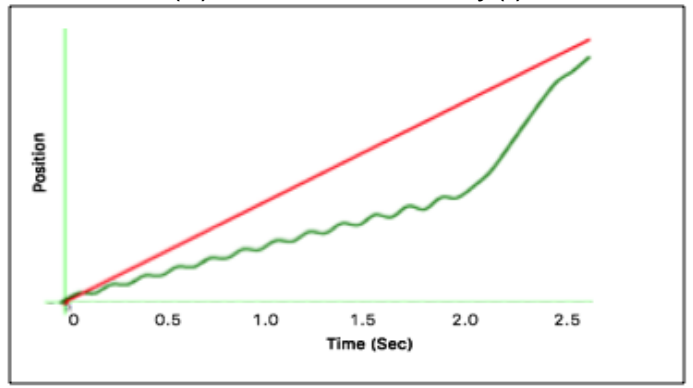

(d) Posture error of DDMR

Figure 9. Steady state errors of trajectory tracking with FLC 2 


\section{Case 3: FLC with 3 MFs, where inputs of MFs were bound by $[-4,4]$ and outputs of MFs} were bound by $[0,6]$

The interval of fuzzy sets was changed from $[-1,1]$ to $[-4,4]$ in the third experiment. As a result, the bound of uncertainty for inputs of MFs described the imprecision and noise within the sensors. Moreover, as every instrumentation element possesses uncertainties from the gathered data; sensors, amplifiers, analog to digital converters (ADC), digital to analog (DAC), and actuators are applied to overcome such error [35]. Furthermore, the control actions, which result from the change of the actuators, could derive from the inconsistencies generated by environmental changes [36].

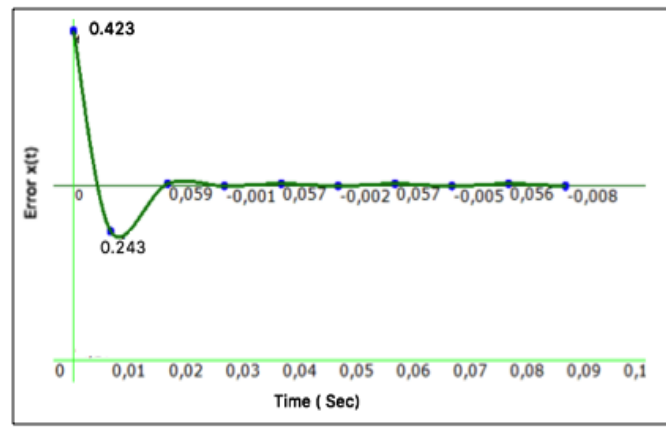

(a) Position error of $\mathrm{x}(\mathrm{t})$

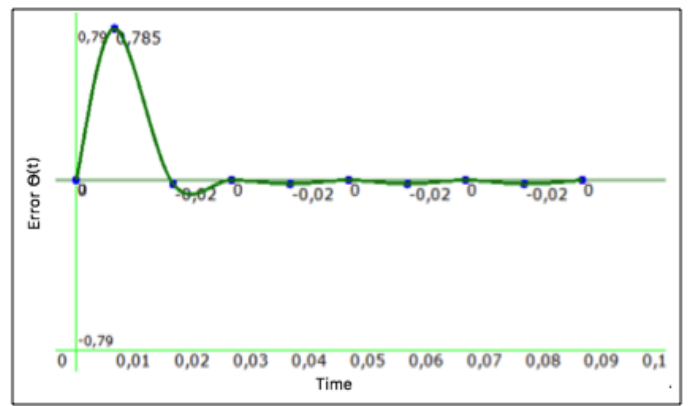

(c) Orientation error of $\theta(t)$

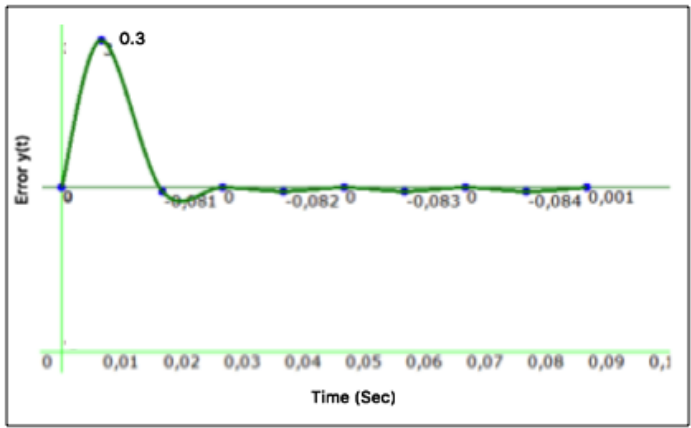

(b) Position error of $y(t)$

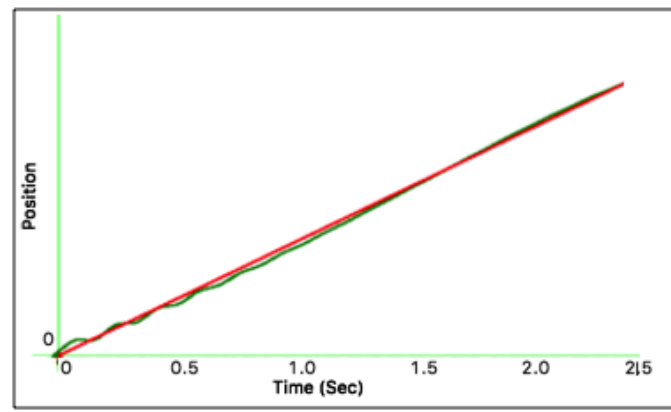

(d) Posture error of DDMR

Figure 10. Steady state errors of trajectory tracking FLC 3

Figure $10(a),(b)$, and (c) present both position and orientation errors. In comparison to experiment 1 , the use of interval of MFs input at $[4,4]$ while retaining MFs output decreased the response of error trajectory to zero within a short time by about $0.09 \mathrm{sec}$ for error position and about $0.08 \mathrm{sec}$ for orientation error. In addition, the trajectory tracking had been closer to reference, as exhibited in Figure 10(d). Therefore, one can note that in experiment 3, the fuzzy controller generated successful solutions for systems tainted with inherent imprecision primarily because robustness was present in the face of imprecision. Furthermore, by applying fuzzy sets as universe of discourse; $[-4,4]$ and $[0,6]$, in MFs input and output, the fuzzy controller displayed the capability of managing the tracking error and successfully applying this capability in trajectory control of non-holonomic mobile robot to be close to zero. In fact, the results showed that the steady state errors had been approximately $1.08 \%, 0.02 \%$, and $0.16 \%$ for $x(t), y(t)$, and $\theta(t)$, respectively. Besides, the actual error of the trajectory to the reference was about $0.0002 \%$, indicating the ability of the controller to force the steady state error to the desired $0 \%$.

Case 4: FLC with 3 MFs, where inputs of MFs were bound by $[-4,4]$ and outputs of MFs were bound by $[0,8]$

In mobile robot control, the terms 'precision' and 'accuracy' are often interchangeable mainly because the degree of certainty derives from measurement. Therefore, the percentage 
of measurement uncertainty only reflects an estimated value. As for experiment 4 , the interval of MFs output was modified from $[0,6]$ to $[0,8]$ to identify the imprecision in the actuator while executing action from the fuzzy controller. As such, the simulation results when the interval of uncertainty was changed into a range of $[0,8]$ are displayed in Figures 11 (a)-(c), whereas the tracking error position of the mobile robot is illustrated in Figure 11(d). Nevertheless, the system turned unstable for certain values.

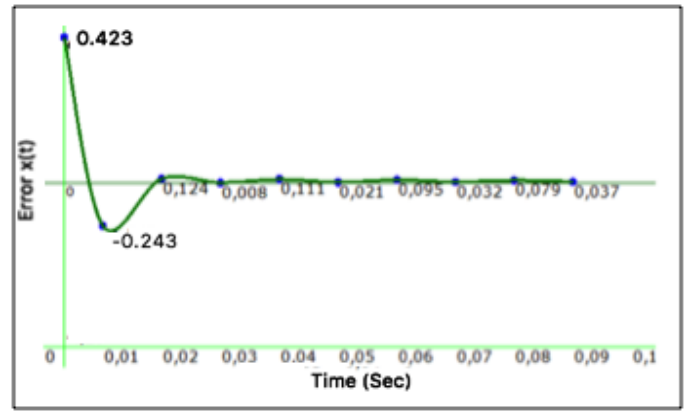

(a) Position error of $x(t)$

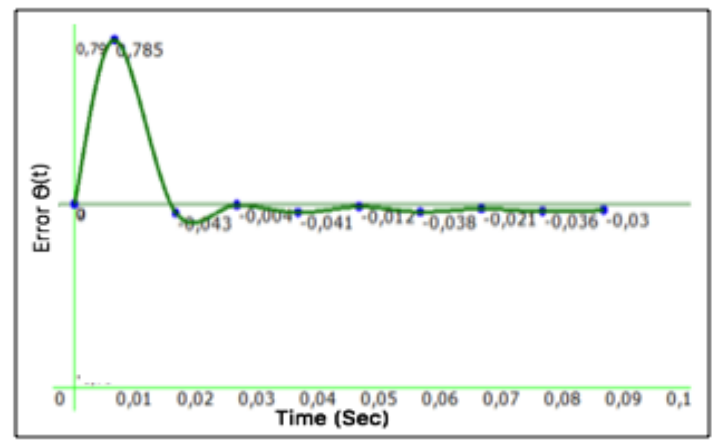

(c) Orientation error of $\theta(t)$

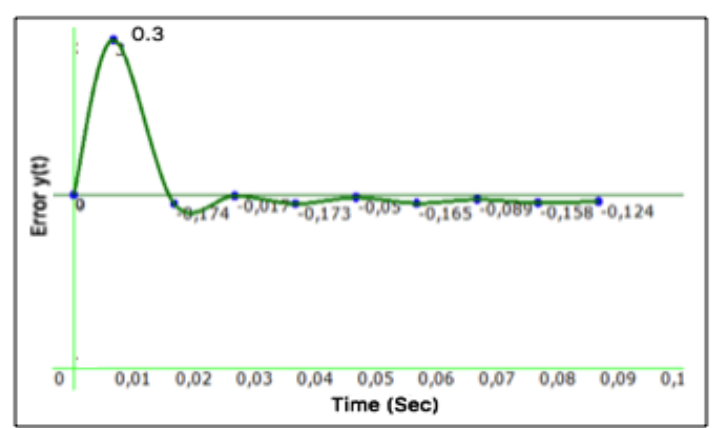

(b) Position error of $\mathrm{y}(\mathrm{t})$

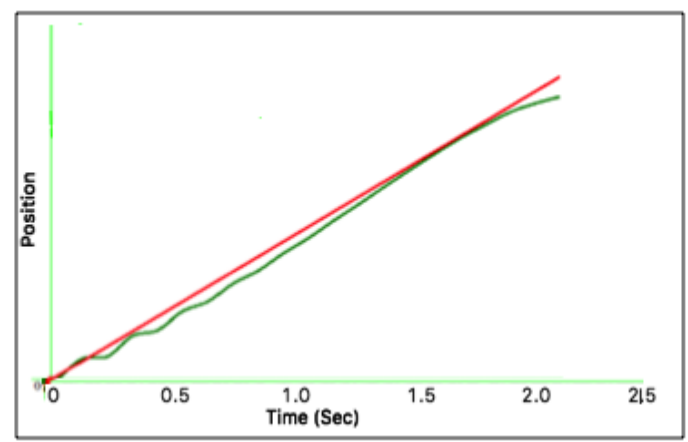

(d) Posture error of DDMR

Figure 11. Steady state errors of trajectory tracking with FLC 4

Figure 11 shows that the time response of both position and orientation errors failed to decrease to zero value, as the steady state errors were found to retain at the end of the graphics. Furthermore, the trajectory error value decreased for a short time, but it became unstable after that. This was because the interval of the MFs output had been too large, thus had become less appropriate.

In this study, the changes of error, $\Delta e(t)$, for position and orientation functioned as inputs for fuzzy controller, $\Delta e(t)$, in which small changes were determined for varied errors. Based on Equation (15), the difference in errors had been calculated to determine the speed of change for each position and orientation error in obtaining point of reference. Besides, Figure 12 summarized all $\Delta e(t)$ for $\mathrm{x}(\mathrm{t}), \mathrm{y}(\mathrm{t})$, and $\theta(t)$ towards the zero value within a short time, which was under $0.05 \mathrm{sec}$.

In order to evaluate the proposed approach upon non-holonomic system, several experiments had been carried out to display the performance of fuzzy controller by using simulation. In fact, Table 2 depicts the variation for universe of discourse to determine the universe of discourse as values for they can give coarse or fine control effects. Hence, a rule set for fuzzy had been developed based on these variables, which had been utilized to modify the fuzzy controller parameters precisely to identify the differences of parameters within the system. These error responses were determined to exemplify the efficiency and the success of the proposed system. It also showed the time response and the steady state error when such controller brought the values of position and orientation errors closer to the values of trajectory reference. In addition, Table 2 portrays that when the universe of discourse was changed, the 
time response also changed in terms of overshoot, rise time, and steady state error. Furthermore, as depicted in experiment 4 with universe of discourse at $[-4,4]$ and $[0,6]$, the response exhibited a good performance, in comparison to other experiments. This is because, experiment 4 had successfully generated small errors $(x=0.1085, y=0.0002$ and $\theta=0.0016)$ and steady state error closer to the desired value; zero. However, the time to produce such value was not satisfactory as it was not rapid. Therefore, more studies are needed to combine the fuzzy controller with the Particle Swarm Optimization (PSO) approach in order to optimize the values of universe of discourse.

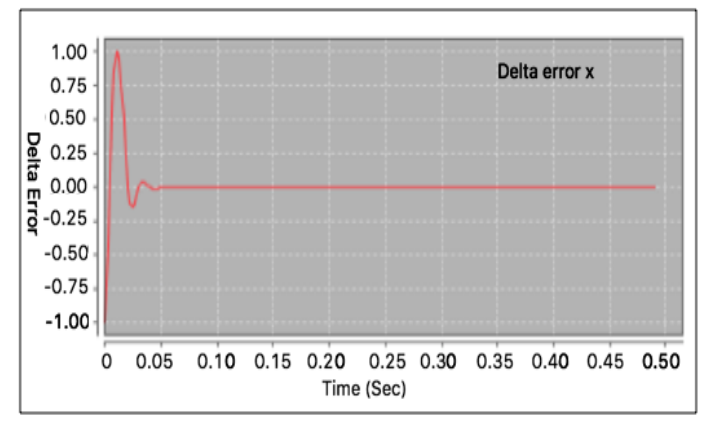

(a) Change of error for $x(t)$ position

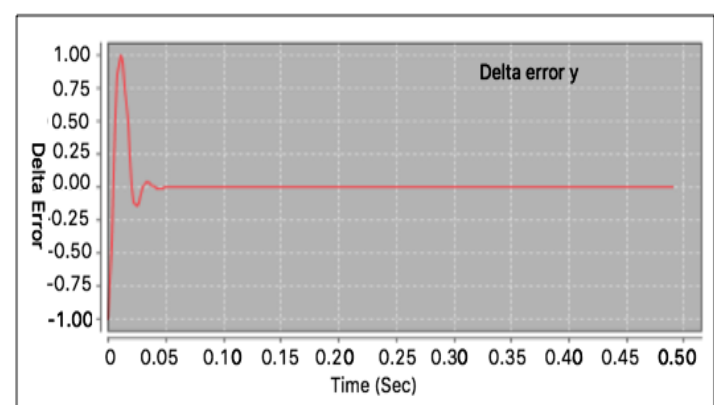

(b) Change of error for $y(t)$ position

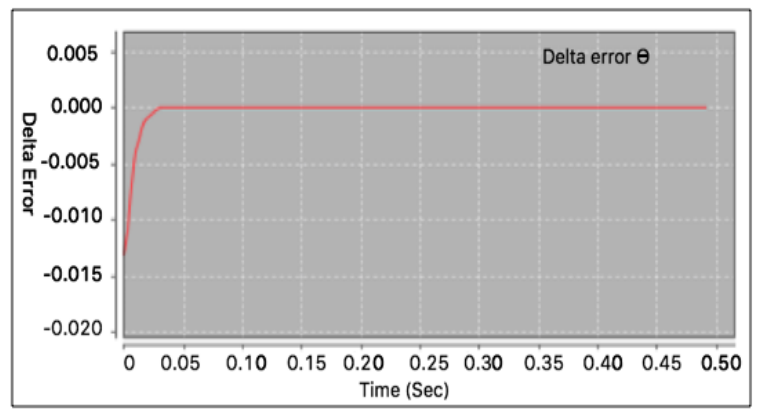

(c) Change of error for $\theta(t)$ orientation

Figure 12. Changes in error values $(\Delta e(t))$ in response of $[-4,4]$ MFs input and $[0,6]$ MFs output

Table 2. The error trajectory with some universe of discourse

\begin{tabular}{cccccccc}
\hline No & $\begin{array}{c}\text { Universe of Discourse } \\
\text { Input } \\
\text { MFs }\end{array}$ & $\begin{array}{c}\text { Output } \\
\text { MFs }\end{array}$ & \multicolumn{2}{c}{$\begin{array}{c}\text { Error average of position and } \\
\text { orientation }\end{array}$} & \multicolumn{3}{c}{ Time response average } \\
(sec)
\end{tabular}

\section{Conclusion}

A tracking controller with fuzzy logic controller of, along with automatic tuning for universe of discourse, for non-holonomic mobile robot is proposed. The design of the fuzzy controller incorporated two significant phases: knowledge-based design and universe of discourse tuning. The systematic procedure involved in developing the knowledge-based design and tuning of universe of discourse had actually applied a rather simple strategy. In fact, the rules are normally extracted from practical experience, which may generate rather subjective results. Moreover, it is also difficult to design a database due to a number of uncertainties that 
had to be taken into account. As such, this study had adopted the universe of discourse as uncertainties for both input and output MFs, which had been generated automatically with some intervals. It also described the function of fuzzy controller in managing the values of $v(t)$ and $\omega(t)$ in the mobile robot to force the position $\left(e_{x}, e_{y},\right)$ and orientation $\left(e_{-} \theta\right)$ error values towards zero within a short time. In future work, the particle swarm optimization (PSO) approach will be applied to tune the fuzzy MFs, especially to estimate the interval of uncertainty value towards producing good results in tracking control application in terms of accuracy and travel time. As for this study, the simulation results retrieved displayed that the fuzzy logic controller did exemplify good performance in tracking both position and orientation errors to the reference values. As such, the proposed controller had achieved the objective outlined in this study. The error positions $\mathrm{x}(\mathrm{t}), \mathrm{y}(\mathrm{t})$, and $\theta(\mathrm{t})$ had been approximately $1.00 \%, 0.02 \%$, and $0.16 \%$, respectively, while the error for trajectory was about $0.0002 \%$ in adhering the reference with the time taken to track the error to zero at precisely $17 \mathrm{sec}$.

\section{Acknowledgements}

The authors would like to thank Universitas Sriwijaya and Kementrian Riset Teknologi dan Pendidikan Tinggi, Indonesia for their support in our research work, with grant Unggulan Perguruan Tinggi 2017.

\section{References}

[1] Safwan M, Uddin V, Asif M. Non-holonomic Mobile Robot Trajectory Tracking using Hybrid Controller. Mehran University Research Journal of Engineering \& Technology. 2016; 35.

[2] Wang G, Wang C, Song X, Du Q. Trajectory Tracking of Non-holonomic Mobile Robots via DiscreteTime Sliding Mode Controller Based on Un-calibrated Visual Servoing. Computational Intelligence, Networked Systems and Their Applications. 2014: 342-350.

[3] Maalouf E, Saad M, Saliah H. A higher-level path-tracking controller for a four-wheel differentially steered mobile robot. Robotics and autonomous systems. 2006; 54(1): 23-33.

[4] Antonelli G, Chiaverini S. A fuzzy-logic- based approach for mobile robot path tracking. IEEE transactions on fuzzy systems. 2007; 15(2): 211-221.

[5] Tzafestas SG, Deliparaschos KM, Moustris GP. Fuzzy logic path tracking control for autonomous non- holonomic mobile robots: Design of System on a Chip. Robotics and autonomous systems. 2010; 58(8): 1017-1027.

[6] Khooban MH. Design an intelligent proportional-derivative (PD) feedback linearization control for nonholonomic-wheeled mobile robot. Journal of Intelligent \& Fuzzy Systems. 2014; 26(4): 1833-1843.

[7] Murray RM, Walsh G, Sastry SS. Stabilization and tracking for non-holonomic control systems using time-varying state feedback. IFAC Nonlinear control systems design. 2016: 109-114.

[8] Chatterjee A, Chatterjee R, Matsuno F, Endo T. Augmented Stable Fuzzy Control for Flexible Robotic Arm Using LMI Approach and Neuro-Fuzzy State Space Modeling. IEEE Transaction on Industrial Electronics. 2008; 55(3): 1256-1270.

[9] Zhifei C, Aghakhani S, Man J, Dick S. ANCFIS: A Neurofuzzy Architecture Employing Complex Fuzzy Sets. IEEE Transaction on Fuzzy Systems. 2011; 19(2): 305-322.

[10] Precup RE, Rădac MB, Tomescu ML, Petriu EM, Preitl S. Stable and convergent iterative feedback tuning of fuzzy controllers for discrete-time SISO systems. Expert Systems with Applications. 2013; 40: 188-199.

[11] Hušek P, Cerman O. Fuzzy model reference control with adaptation of input fuzzy sets. KnowledgeBased Systems. 2013; 49: 116-122.

[12] Antonelli G, Chiaverini S. A fuzzy-logic- based approach for mobile robot path tracking, IEEE transactions on fuzzy systems. 2007; 15(2): 211-221.

[13] Herbert G, Tanner ISS. Properties of Non-holonomic Mobile Robots. Proceeding of the IEEE/RSJ Intl. Conf on Intelligent Robots and Systems. Las Vegas. 2003: 3799-3804.

[14] El Hajjaji A, Bentalba S. Fuzzy path tracking control for automatic steering of vehicles. Robotics and Autonomous Systems. 2003; 43(4): 203-213.

[15] Tae-Kyeong Yeu, Soung-Jea Park, Sup Hong, Hyung- Woo Kim, Jong-Su Choi. Path tracking using vector pursuit algorithm for tracked vehicles. SICE-ICASE International Joint Conference. Korea. 2006: 2781-2786.

[16] Guilherme V Raffo, Guilherme K Gomes, Julio E Normey-Rico. A Predictive Controller for Autonomous Vehicle Path Tracking. IEEE Transactions on Intelligent Transportation Systems. 2009; 10(1): 92-102.

[17] Fukao T, Nakagawa $\mathrm{H}$, Adachi N. Adaptive tracking control of a non-holonomic mobile robot. IEEE Transactions on Robotics and Automation. 2000; 16(5): 609-615. 
[18] Dixon WE, de Queiroz MS, Dawson DM, Flynn TJ. Adaptive tracking and regulation of a wheeled mobile robot with controller/update law modularity. IEEE Transactions on Control Systems Technology. 2004: 12(1); 138-147.

[19] Jian Wang, Xiangyang Zhu, Masahiro Oya, Chun-Yi Su, Robust motion tracking control of partially non-holonomic mechanical systems. Robotics and Autonomous Systems. 2006; 54(4): 332-341.

[20] Cao Z, Zhao Y, Wu Q. Adaptive Trajectory Tracking Control for a Non-holonomic Mobile Robot. Chinese Journal of Mechanical Engineering. 2011; 24(4): 546-556.

[21] Hou ZG, Zou AM, Cheng L, Tan M. Adaptive Control of an Electrically Driven Non-holonomic Mobile Robot via Backstepping and Fuzzy Approach. IEEE Transactions on Control Systems Technology. 2009; 17(4): 803-815.

[22] Khooban MH. Design an Intelligent Proportional- Derivative (PD) Feedback Linearization Control for Non-holonomic-Wheeled Mobile Robot. Journal of Intelligent and Fuzzy Systems. 2014; 26(4): 18331843.

[23] Wang G, Wang C, Song X, Du Q. Trajectory Tracking of Non-holonomic Mobile Robots via DiscreteTime Sliding Mode Controller Based on Uncalibrated Visual Servoing. In International Conference on Life System Modeling and Simulation and International Conference on Intelligent Computing for Sustainable Energy and Environment. 2014: 342-350.

[24] Mobayen S. Fast Terminal Sliding Mode Tracking of Non-holonomic Systems with Exponential Decay Rate. IET Control Theory \& Applications. 2015; 9(8): 1294-1301.

[25] Mobayen S. Finite-time Tracking Control of Chained-form Non-holonomic Systems with External Disturbances based on Recursive Terminal Sliding Mode Method. Nonlinear Dynamics. 2015; 80: 12.

[26] Mobayen S. A Novel Global Sliding Mode Control Based on Exponential Reaching Law for a Class of Underactuated System with External Disturbances. J. Comput. Nonlinear Dynamic. 2015; 11(2): 9.

[27] Mobayen S. Design of LMI-based Sliding Mode Controller with an Exponential Policy for a Class of Underactuated System. Wiley Periodicals, Inc. Complexity. 2016; 21: 117-124.

[28] Bayat F, Mobayen S, Javadi S. Finite-time Tracking Control of nth-order Chained-form Nonholonomic System in the Presence of Disturbances. ISA Transaction. 2016; 63.

[29] Mobayen S, Javadi S. Disturbance Observer and Finite-time Tracker Design of Disturbed Third-order Non-holonomic System using Terminal Sliding Mode. Journal of Vibration and Control. 2016; 23(2).

[30] Mobayen S, Yazdanpanah MJ, Majd VJ. A Finite-time Tracker for Non-holonomic System using Recursive Singularity Free FTSM. In Proceeding of the American Control Conference. 2011.

[31] Jiangdagger ZP, Nijmeijer H. Tracking Control of Mobile Robots: A Case Study in Backstepping. Automatica. 1997; 33(7): 1393-1399.

[32] Klanèar G, Škrjanc I. Tracking-Error Model- Based Predictive Control for Mobile Robots in Real Time. Robotics and Autonomous Systems. 2007; 55(6): 460-469.

[33] Fukao T, Nakagawa H, Adachi N. Adaptive Tracking Control of a Non-holonomic Mobile Robot. IEEE Transactions on Robotics and Automation. 2000; 16(5): 609-615.

[34] Ren W, Sun JS, Beard R, McLain T. Experimental validation of an autonomous control system on a mobile robot platform. IET Control Theory \& Applications. 2007; 1(6): 1621-1629.

[35] Xuan H, Zeng W. Design of Visual Feedback Tracking Algorithm for Non-holonomic Mobile Robots Based on Neural Network. Sensors \& Transducers. 2013; 157(10): 42-52.

[36] Wai RJ, Liu C. M. Design of dynamic petri recurrent fuzzy neural network and its application to pathtracking control of non-holonomic mobile robot. IEEE Transactions on Industrial Electronics. 2009; 56(7): 2667-2683.

[37] Peri VM, Simon D. Fuzzy logic control for an autonomous robot. In Fuzzy Information Processing Society, NAFIPS. Annual Meeting of the North American. 2005: 337-342.

[38] Oh SY, Park DJ. Self-tuning fuzzy controller with variable universe of discourse. In Systems, Man and Cybernetics, Intelligent Systems for the 21st Century, IEEE International Conference on. 1995; 3: 2628-2632.

[39] Long Z, Liang X, Yang L. Some approximation properties of adaptive fuzzy systems with variable universe of discourse. Information Sciences. 2010; 180(16): 2991-3005.

[40] Wang J, Qiao GD, Deng B. Observer-based robust adaptive variable universe fuzzy control for chaotic system. Chaos, Solitons \& Fractals. 2005; 23(3): 1013-1032.

[41] Mendel JM. Fuzzy logic systems for engineering: a tutorial. Proceedings of the IEEE. 1995; 83(3): 345-377.

[42] Nurmaini S, Zaiton S, Norhayati D. An embedded interval type-2 neuro-fuzzy controller for mobile robot navigation. In Systems, Man and Cybernetics. SMC IEEE International Conference on. Texas. 2009: 4315-4321.

[43] Patricia M, Castillo O. A review on type-2 fuzzy logic applications in clustering, classification and pattern recognition. Applied soft computing. 2014; 2: 568-577. 